\title{
REA-UML: Recurso Educacional Aberto para Ensino da UML
}

\author{
Jonas Piccin Laroza, Rodrigo Duarte Seabra \\ Instituto de Matemática e Computação - Universidade Federal de Itajubá (UNIFEI) \\ Caixa Postal 50 - 37.500-903 - Itajubá - MG - Brasil \\ jonaspiccinlaroza@gmail.com, rodrigo@unifei.edu.br
}

\begin{abstract}
Teaching UML is a complex process. The traditional teaching methodology guided only by lectures is not characterized as the most appropriate strategy to teach modeling concepts so that students successfully learn the contents and the good software engineering practices involved in this activity. The study proposes using a tool that will contribute to stimulating the apprentice in pursuit of knowledge concerning the UML, presenting a new approach in teaching the topic through an open educational resource named REA-UML. The main results showed that the tool proposed is a good alternative to be used in the classroom contributing to learning the theme.
\end{abstract}

Resumo. $O$ ensino da UML é um processo complexo. A metodologia tradicional de ensino pautada apenas por aulas expositivas não se caracteriza como a estratégia mais adequada para ensinar conceitos de modelagem de forma que os estudantes assimilem com exito o conteúdo e as boas práticas de engenharia de software envolvidas nesta atividade. $O$ estudo propõe o uso de uma ferramenta que contribuirá para o incentivo do aprendiz na busca do conhecimento relativo à UML, apresentando uma nova abordagem no ensino do tema por meio de um recurso educacional aberto denominado REA-UML. Os principais resultados mostraram que a ferramenta proposta se apresenta como uma boa alternativa a ser explorada em sala de aula contribuindo para a aprendizagem do tema.

\section{Introdução}

A inovação no setor tecnológico tem crescido de forma que a sociedade já não se vê sem o uso de recursos tecnológicos em seu cotidiano (Nicolaci da Costa 2002). À medida que essa inovação cresce e diversas tarefas tornam-se automatizadas, faz-se necessário desenvolver softwares que atendam aos requisitos dos usuários. Para isso, deve-se conceber uma arquitetura sólida que permita modificações. Visando a alcançar esse objetivo, a modelagem do sistema deve ser bem feita de modo a transparecer ao desenvolvedor as características e o comportamento do produto final.

A modelagem é a etapa fundamental dentre as atividades que levam à implantação de um software robusto. Ao considerar que um modelo representa uma abstração da realidade, um sistema computacional pode ser representado em função de variados aspectos, com a utilização de diferentes modelos (Booch, Rumbaugh e Jacobson 2012). No que concerne ao desenvolvimento de software, a modelagem é usada para representar as especificações de um sistema real por meio de diagramas, permitindo a compreensão das funcionalidades e comportamentos do software antes de implementá-lo (Furgeri 2013). Desde a década de 80, vários métodos eram utilizados para a modelagem orientada a objetos, até a unificação da UML - Unified Modeling Language - com a junção dos estudos de Booch, Rumbough e Jacobson (Larman 2007). 


\section{CBIE-LACLO 2015}

Anais do XXVI Simpósio Brasileiro de Informática na Educação (SBIE 2015)

Portanto, a UML é uma linguagem unificada para a modelagem orientada a objetos.

Devido a sua importância torna-se fundamental o ensino da UML nos cursos de computação e afins. Contudo, as metodologias adotadas no ensino superior costumam, em sua maioria, se caracterizar por aulas expositivas e dialogadas. Os conceitos são apresentados sem a preocupação de relacioná-los a um contexto válido ou ambientá-los com conceitos iniciais, e na forma como os diferentes diagramas se completam (Tanaka 2011). Diante deste cenário, o aprendizado ainda ocorre de modo mecanizado, menosprezando conceitos sobre a importância da modelagem.

Estudos apontam que o ensino da UML é complexo e que a maioria dos projetistas encontra obstáculos relacionados às suas dificuldades cognitivas (Flint, Gardner e Boughton 2004; Kuzniarz e Börstler 2011; Bera 2012). Por se tratar de uma área que requer elevado poder de abstração, a metodologia comumente explorada para $\mathrm{o}$ ensino do tema não estimula a aprendizagem do aluno, portanto, segundo a visão de Paiva e Medeiros (2011), deve ser criado um modelo que incentive o estudo e a compreensão dos termos e conceitos necessários para desenvolver uma modelagem que reflita a realidade, algo que apenas um bom projetista é capaz de garantir. Ressalta-se, neste momento, que este profissional é muito requisitado na indústria do software.

A partir do cenário exposto, esta pesquisa tem como objetivo geral apresentar o desenvolvimento de uma ferramenta que, possivelmente, privilegie o entendimento da modelagem UML por meio de recursos educacionais abertos, disponíveis na Internet, superando as limitações geográficas e usufruindo de abordagens amplamente utilizadas em cursos massivos online. Adicionalmente, o trabalho ainda se propõe a investigar, sob a ótica discente, os potenciais benefícios apontados em decorrência do uso da ferramenta proposta no apoio ao ensino da UML. Como principais contribuições do estudo destacam-se: (i) contribuir com a disseminação da ideia do uso de recursos educacionais abertos para que futuramente haja aumento de sua utilização na esfera educacional; (ii) avaliar qualitativamente a opinião dos estudantes envolvidos no estudo verificando se a ferramenta desenvolvida nesta oportunidade atendeu as expectativas esperadas, bem como seu grau de aceitação e contribuição no processo de ensino.

Nesse sentido, a seção 2 discute a importância da UML para a modelagem de processos e sistemas computacionais, bem como alguns trabalhos correlatos. A seção 3 discorre sobre a ferramenta desenvolvida neste estudo e o método empregado em sua avaliação. A seção 4 apresenta a análise dos dados coletados na pesquisa experimental realizada e, finalmente, a seção 5 apresenta as conclusões do trabalho.

\section{Fundamentação Teórica}

A UML é uma linguagem visual usada para representar sistemas por meio de diferentes diagramas, isto é, retrata a modelagem de softwares com base em variadas perspectivas, facilitando a comunicação dos profissionais envolvidos no desenvolvimento de um sistema (Guedes 2011). Essa linguagem padrão foi concebida, sobretudo, para o desenvolvimento de sistemas orientados a objetos. Em sua versão atual é composta por treze diagramas, classificados em estruturais ou comportamentais. Além disso, como toda linguagem, a UML possui vocabulário e um conjunto próprio de regras.

Os diagramas UML permitem melhor visualização do sistema, sua arquitetura e componentes, e principalmente os relacionamentos entre eles, bem como as especificações do sistema e sua documentação (Melo 2010). Por essas vantagens, a 


\section{CBIE-LACLO 2015}

Anais do XXVI Simpósio Brasileiro de Informática na Educação (SBIE 2015)

UML é usada não apenas para a modelagem de software, mas também apresenta grande potencial para modelar outras tarefas, arquiteturas e fluxos de trabalho diversos, com a finalidade de simplificar a realidade por meio de representações padronizadas.

Devido ao fato de a UML ser uma linguagem de modelagem mundialmente utilizada, várias pesquisas demonstram preocupação com seu ensino e aprendizagem. Nos cursos de computação, em geral, o tema é abordado na disciplina de engenharia de software, apesar de existirem casos em que o assunto é tratado em disciplinas específicas direcionadas ao ensino dessa linguagem. No entanto, muitas vezes aulas expositivas e dialogadas se tornam mecanizadas e não são suficientes para a aprendizagem completa do conteúdo.

Para Santana, Rossini e Pretto (2012), o modelo de educação ainda atualmente empregado (um professor para muitos alunos) é apenas uma das possíveis configurações de ensino-aprendizagem existentes. Com a crescente popularização da internet, muitos especialistas acreditam que o modelo de educação atual está em um processo de falência, pois os estudantes fazem parte de uma geração digital que não aceita mais esse modelo rígido. Nesse cenário, o desenvolvimento de mídias digitais proporcionou um impulso para novas configurações de ensino, e a criação de recursos, espaços e tecnologias abertas pode impulsionar inúmeras oportunidades educacionais. Portanto, os Recursos Educacionais Abertos (REA) fazem parte dessa expansão e são propulsores de novas configurações de ensino-aprendizagem. Na prática, a definição apresentada por Santos (2013) deixa claro o papel de um REA ao afirmar que são materiais de ensino, aprendizagem ou pesquisa que estejam em domínio público ou que tenham sido disponibilizados sob uma licença de propriedade intelectual que permita seu livre uso e adaptação por terceiros.

Ademais, segundo Flint, Gardner e Boughton (2004), a complexidade da UML inibe o ensino de toda sua abrangência. Desse modo, encontram-se registradas na literatura científica variadas pesquisas focadas no ensino da UML de maneira diferenciada, visando a proporcionar uma aprendizagem significativa e complementar às atividades realizadas em sala de aula.

\subsection{Trabalhos Correlatos}

Grützner e Bunse (2002) propuseram uma abordagem de ensino misto, resumindo as vantagens provenientes da modalidade presencial com a online. $\mathrm{Na}$ ocasião, o curso foi ministrado inicialmente na modalidade online, com o objetivo de nivelar o conhecimento dos estudantes. Em um segundo momento, a partir da homogeneidade da turma, o curso foi ministrado de modo presencial, o que, segundo os autores do estudo, permitiu melhor aproveitamento das aulas.

No âmbito da colaboração, Paiva e Medeiros (2011) defenderam uma proposta de abordagem pautada por esse paradigma. Para tanto, desenvolveram a metodologia ESCollab, com a finalidade de tornar o ensino de engenharia de software mais interativo e atraente aos aprendizes explorando recursos da web 2.0. Dividida em três fases, cada qual com entradas, saídas e participantes, a aplicação da metodologia proposta apresentou bons resultados, ressaltando o valor do gerenciamento e a cultura de cooperação entre os agentes do processo de ensino-aprendizagem da engenharia de software.

Com uma abordagem educacional direcionada para deficientes visuais, o trabalho de Silva, Pansanato e Fabri (2010) defende a premissa de que a inserção de estudantes 


\section{CBIE-LACLO 2015}

Anais do XXVI Simpósio Brasileiro de Informática na Educação (SBIE 2015)

cegos em cursos de computação é uma tarefa desafiadora. Conforme a opinião dos autores, isso se deve às inúmeras dificuldades inerentes às técnicas de ensino e aprendizagem que estão despreparadas para essa realidade. $\mathrm{O}$ estudo propõe o uso de uma tabela como uma representação alternativa para os diagramas da UML, permitindo assim que o estudante com deficiência visual possa compreender e construir um diagrama sem o auxílio de outro indivíduo ou, até mesmo, de qualquer equipamento especial. O resultado do estudo apontou que a técnica é eficiente e que o trabalho é uma contribuição importante para a educação superior em computação, possibilitando a inclusão de estudantes com deficiências visuais nas disciplinas que envolvem a modelagem de sistemas. Outro estudo recente direcionado a esse público é a pesquisa de Luque et al. (2014).

Observando a dificuldade dos estudantes em assimilar os conceitos da UML e a dos professores em ministrar o conteúdo relativo ao tema, Tanaka (2011) elaborou mapas conceituais e recursos de workflow para auxiliar o ensino-aprendizagem de alguns diagramas. No referido estudo foi elaborado um mapa conceitual e um workflow para cada tipo de diagrama abordado, com o objetivo de orientar a modelagem de modo que os aprendizes pudessem compreender os conceitos nela envolvidos. A aplicação dessa metodologia permitiu contextualizar o aluno em relação ao assunto abordado, garantindo melhor aproveitamento do conteúdo.

A pesquisa de Rodrigues (2012) apresenta a abordagem VisAr3D - Visualização de Arquitetura de Software em 3D - desenvolvida como uma proposta inovadora com o objetivo de proporcionar a exploração e interação com modelos UML por meio da visualização tridimensional. Na pesquisa, diagramas 3D são gerados automaticamente a partir de um diagrama bidimensional existente, fornecendo uma semântica mais rica para que os estudantes compreendam os elementos envolvidos na modelagem, bem como suas relações.

Cera, Forno e Vieira (2012) acreditam que a metodologia mais indicada para o ensino-aprendizagem da UML consiste no Aprendizado Baseado em Problemas - ABP que permite aproximar teoria e prática por meio de discussões entre pequenos grupos visando a solucionar um problema. A metodologia foi aplicada durante seis períodos, dividindo os alunos em grupos de trabalho para desenvolver um sistema computacional que solucionasse um desafio. O objetivo consistiu em estimular não apenas os conhecimentos de engenharia de software, mas também aspectos relacionados ao cotidiano profissional.

A partir das pesquisas arroladas, é fato que o estudo da UML tem despertado o interesse de pesquisadores no desenvolvimento de projetos diferenciados que facilitem o ensino e a aprendizagem do tema. Na mesma direção dos estudos apresentados, o fator motivador envolvido nesta oportunidade também consiste em proporcionar apoio aos estudantes que, muitas vezes, demonstram ou relatam algum tipo de dificuldade, seja na compreensão ou na realização da modelagem UML. Assim, o presente trabalho se apresenta como mais uma alternativa frente a esse desafio, abrangendo uma nova metodologia para o estudo da UML.

\section{Método}

\subsection{A Ferramenta REA-UML}

A partir de uma família de aplicações genéricas de hipermídia online concebida por 


\section{CBIE-LACLO 2015}

Anais do XXVI Simpósio Brasileiro de Informática na Educação (SBIE 2015)

Kirner (2013) e batizada de RE-MIIO ${ }^{1}$ (Recurso Educacional com Mídias Interativas Integradas Online), o material derivado e apresentado nesta oportunidade, REA-UML (Recurso Educacional Aberto para Ensino da UML) ${ }^{2}$, é composto por elementos de textos, sons (no formato de narrações), imagens, vídeos e páginas web.

Com base na estrutura padrão do RE-MIIO, tem-se um objeto raiz representado por uma imagem centralizada na tela e, a partir dela, o usuário pode acessar conteúdos disponíveis em agrupamentos, no formato de menus, que apresentam detalhes com base na preferência do criador do objeto de aprendizagem. $\mathrm{O}$ usuário pode acionar recursos multimídia associados aos agrupamentos por meio de um processo simplificado de interação. Pesquisas recentes envolvendo a produção de outras adaptações elaboradas a partir do RE-MIIO podem ser encontradas em Silva (2013), Seabra, Romano e Oliveira (2014) e Torres (2014). Em se tratando do estudo da UML, o REA-UML, objeto de estudo em questão, não explorou recursos de imagens 3D e realidade aumentada disponíveis na ferramenta original, pois, segundo os autores desta pesquisa, os referidos recursos não agregariam na compreensão dos conteúdos produzidos. A Figura 1 apresenta a implementação do REA-UML sob a forma de uma página na internet, com parte de seus recursos interativos visíveis.

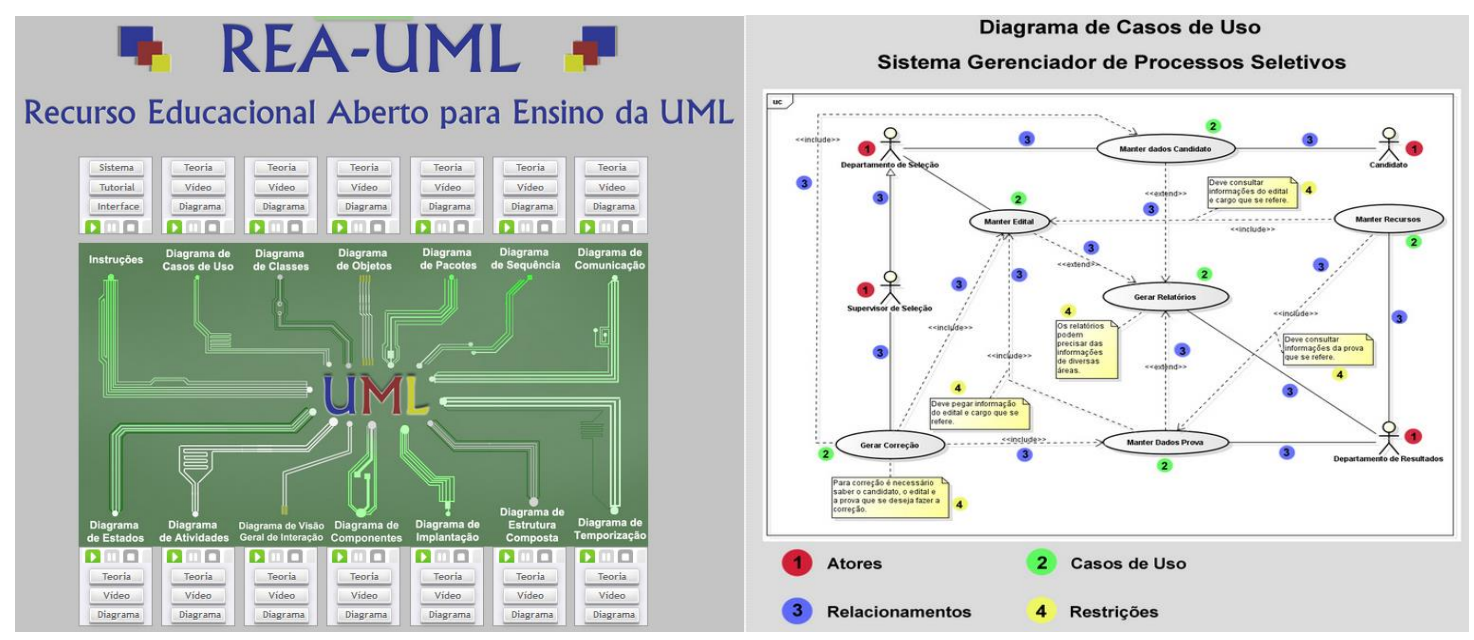

Figura 1- Implementação do REA-UML. A imagem à esquerda ilustra a interface geral do ambiente e, a da direita, apresenta um exemplo de modelagem UML acionado a partir da interface principal.

A estrutura lógica do REA-UML consiste de dois componentes: nó central e agrupamentos de mídias. O nó central apresenta uma imagem ilustrativa visando à organização e divisão dos conceitos representados na interface principal. Nela estão representados catorze agrupamentos, sendo que o primeiro deles objetiva contextualizar informações iniciais aos estudantes, a saber: i) problema proposto, retratando as especificações de uma situação-problema que deverá ser modelada pelo estudante; ii) breve tutorial explicativo sobre o ambiente; iii) imagem com a representação dos principais elementos disponíveis na interface da ferramenta.

A partir do segundo agrupamento, cada grupo explorado na ferramenta desenvolvida diz respeito a um diagrama da UML, totalizando treze diferentes diagramas. Na prática, cada agrupamento é composto por variadas mídias. A partir do

\footnotetext{
${ }^{1} \mathrm{http}: / / \mathrm{www} . c k i r n e r . c o m /$ midias

${ }^{2} \mathrm{http}: / /$ sgvclin.altervista.org/rea-uml/
} 


\section{CBIE-LACLO 2015}

Anais do XXVI Simpósio Brasileiro de Informática na Educação (SBIE 2015)

nó central o usuário tem a liberdade de acessar diferentes conteúdos relacionados a um diagrama específico da UML. Nesse âmbito, as informações disponíveis em cada agrupamento versam sobre: i) conceitos teóricos sobre o diagrama, tais como suas características, função e elementos envolvidos; ii) vídeo tutorial como auxílio para o desenvolvimento do diagrama usando uma ferramenta específica de modelagem UML; iii) a modelagem do diagrama propriamente dito; iv) áudio contendo uma breve explicação do diagrama por meio de narrativa.

Como apoio à condução do processo de ensino da UML, nesta pesquisa, foi explorado um estudo de caso, envolvendo a modelagem de um sistema gerenciador de concursos públicos, adaptado a partir de Melo (2010). A partir dessa metodologia espera-se que os aprendizes absorvam mais facilmente os aspectos lógicos envolvidos na modelagem do sistema proposto.

\subsection{Participantes e Descrição do Método}

Após o desenvolvimento da ferramenta proposta foram oferecidas aulas sobre modelagem de sistemas orientados a objetos por meio da linguagem UML a 37 alunos regularmente matriculados na disciplina de Computação Orientada a Objetos II, do quinto período do curso de Ciência da Computação, da Universidade Federal de Itajubá - UNIFEI. A amostra foi composta por 30 estudantes do sexo masculino e sete do gênero feminino.

O método para a aplicação da pesquisa experimental consistiu de duas etapas. $\mathrm{Na}$ primeira, os alunos foram comunicados quanto à experimentação do REA-UML no processo de ensino, bem como sobre o propósito principal da pesquisa em questão tendo em vista que o possível fator de influência não está no objetivo do trabalho, mas sim, no conhecimento das expectativas dos autores da pesquisa em relação aos potenciais resultados. Após as explicações iniciais, iniciaram-se as aulas propriamente ditas com a apresentação dos conteúdos referentes à disciplina. Ao final de cada aula, os alunos participantes do estudo eram estimulados a exercitarem os conteúdos teóricos vistos em sala de aula na ferramenta REA-UML. Ademais, como a ferramenta proposta se encontra disponível em um repositório aberto na internet, os alunos ainda eram incentivados a utilizá-la fora do ambiente escolar. O processo de intervenção se estendeu por seis semanas.

$\mathrm{Na}$ última semana do estudo, os participantes foram informados sobre a realização de mais uma atividade didática pertencente à segunda etapa da pesquisa, que consistiu em responder um questionário avaliativo sobre o uso da ferramenta. $\mathrm{O}$ questionário consistiu de 10 questões de múltipla escolha visando a minimizar a subjetividade de questões abertas, além de um espaço para descrição de aspectos relevantes caso os alunos tivessem comentários adicionais. A aplicação do questionário levou cerca de 15 minutos e os autores do estudo não interferiram ou ajudaram os participantes em respondê-lo. Os estudantes foram informados sobre o anonimato e uso dos dados coletados apenas para fins de pesquisa. O Quadro 1 apresenta as questões investigadas, além de uma questão final que solicita comentários de ordem geral.

\section{Questões}

Q1. De maneira geral, a utilização do REA-UML foi de fácil compreensão?

Q2. Os recursos visuais disponibilizados pela ferramenta (cores, imagens, botões, dimensão das 


\section{CBIE-LACLO 2015}

Anais do XXVI Simpósio Brasileiro de Informática na Educação (SBIE 2015)

telas etc.) são de boa qualidade?

Q3. A ferramenta apresenta os recursos necessários para o ensino adequado da UML?

Q4. Qual tipo de mídia você mais usufruiu ao utilizar o REA-UML?

Q5. O conteúdo disponível na ferramenta foi de fácil entendimento?

Q6. O uso do REA-UML contribuiu para a aprendizagem do conteúdo trabalhado na disciplina de Computação Orientada a Objetos II?

Q7. Tendo contato com a UML, que percentual de conhecimento foi agregado após o uso do REA-UML?

Q8. O REA-UML tornou mais clara a forma de estudar os diagramas da UML?

Q9. Você gostaria que outras disciplinas explorassem ferramentas similares para o ensino e reforço dos conteúdos aprendidos em sala de aula?

Q10. Em geral, como você classificaria a eficácia do REA-UML no processo de ensino e aprendizagem da UML?

Comentários adicionais:

Quadro 1 - Questões da avaliação qualitativa sobre a utilização do REA-UML.

\section{Análise dos Dados}

A primeira questão da avaliação buscou identificar se o uso do REA-UML era simples e como se deu a compreensão do estudante sobre a ferramenta. Quase a totalidade da amostra respondeu que a compreensão foi fácil, o que corresponde a 95\% (46\% responderam totalmente e $49 \%$ responderam muito) da amostra.

A segunda questão está relacionada à qualidade da interface. Nesse contexto, $78 \%$ (16\% atribuiu excelente e $62 \%$ responderam boa) dos participantes afirmaram que a ferramenta apresenta recursos visuais de boa qualidade, totalizando 29 respostas positivas. Nesta questão, apenas $8 \%$ dos participantes consideraram que a qualidade dos recursos oferecidos é ruim.

A próxima investigação (questão 3) diz respeito à ferramenta oferecer os recursos necessários para o ensino adequado da UML segundo a visão dos estudantes. Neste caso, $75 \%$ (16\% responderam totalmente e 59\% atribuíram muito) dos participantes concordaram que os recursos são suficientes para o ensino da UML. Ainda nesta questão, $22 \%$ da amostra atribuiu avaliação regular e apenas 1 estudante respondeu que a ferramenta contribuiu pouco.

A quarta e quinta questão foram propostas com os seguintes objetivos: verificar qual tipo de mídia os alunos mais utilizaram no REA-UML (questão 4); e mensurar se os conteúdos presentes nessas mídias foram de fácil entendimento (questão 5). A quarta questão não necessariamente envolveu a seleção de apenas uma alternativa, pois os estudantes podem ter explorado mais de uma categoria de mídia com a mesma frequência. Os resultados mostraram que $61 \%$ dos participantes usufruíram mais da mídia imagens em seus estudos. $\mathrm{O}$ acesso às demais mídias apresentou a seguinte distribuição: textos, com $28 \%$ das respostas; vídeos, 9\% das opiniões; e apenas um estudante respondeu áudios. A maioria das opiniões favoráveis às imagens pode ser explicada em função dessa categoria de mídia apresentar exemplos de modelagens a partir dos quais os estudantes puderam utilizar como base para a realização de 


\section{CBIE-LACLO 2015}

Anais do XXVI Simpósio Brasileiro de Informática na Educação (SBIE 2015)

exercícios. Vale destacar que, no contexto desta questão, os estudantes apontaram a categoria de mídia que mais se identificaram como fonte de conhecimento, o que não significa que não tenham explorado as demais categorias. Para a questão 5 , as opiniões foram, em sua maioria, positivas: $35 \%$ dos participantes responderam totalmente; $57 \%$ atribuíram muito; e apenas $8 \%$ destacaram entendimento regular.

Em relação à contribuição do REA-UML no aprendizado do conteúdo trabalhado na disciplina (questão 6), novamente verifica-se resultado positivo, isto é, $70 \%$ da amostra concordou que o uso da ferramenta contribuiu totalmente ou muito no estudo da disciplina. No que tange a esta questão, ressalta-se que nenhum estudante atribuiu como avaliação pouco ou nada, o que leva à conclusão de que a ferramenta alcançou seu objetivo.

A sétima questão foi elaborada com o objetivo de mensurar o conhecimento adicional obtido pelos estudantes a partir do uso do REA-UML. Para isso, o ganho apontado pelo participante foi classificado de 0 a $100 \%$. A maioria das respostas (30 alunos) se concentrou no intervalo de 50 a $80 \%$. Essa distribuição corrobora o potencial exercido pela ferramenta no apoio ao ensino do tema. De acordo com o relato de um dos participantes: "A ferramenta é de grande ajuda para consolidar os conhecimentos passados em sala. Sempre que tenho alguma dúvida na resolução dos exercícios propostos, recorro à ferramenta como apoio e, na maioria das vezes, consigo entender e sanar minhas dúvidas".

No caso da questão 8 - o REA-UML tornou mais clara a forma de estudar os diagramas da UML? - 59\% dos participantes concordaram que a ferramenta se apresenta como um método diferenciado que auxilia na aprendizagem da UML. Apenas um aluno respondeu que a ferramenta deixou pouco clara a forma de estudar os diagramas, enquanto $38 \%$ dos participantes optaram pela resposta regular.

No que compete à questão $9,89 \%$ dos participantes relataram que o uso de ferramentas similares seria de grande proveito no estudo e reforço dos conteúdos ministrados em outras disciplinas (Figura 2). O relato de um estudante, em especial, reforça este resultado: "Achei a ferramenta um meio interessante e eficiente para o estudo em casa e gostaria que outros professores adotassem um método parecido, pois só tem a acrescentar no conteúdo dado em sala de aula".

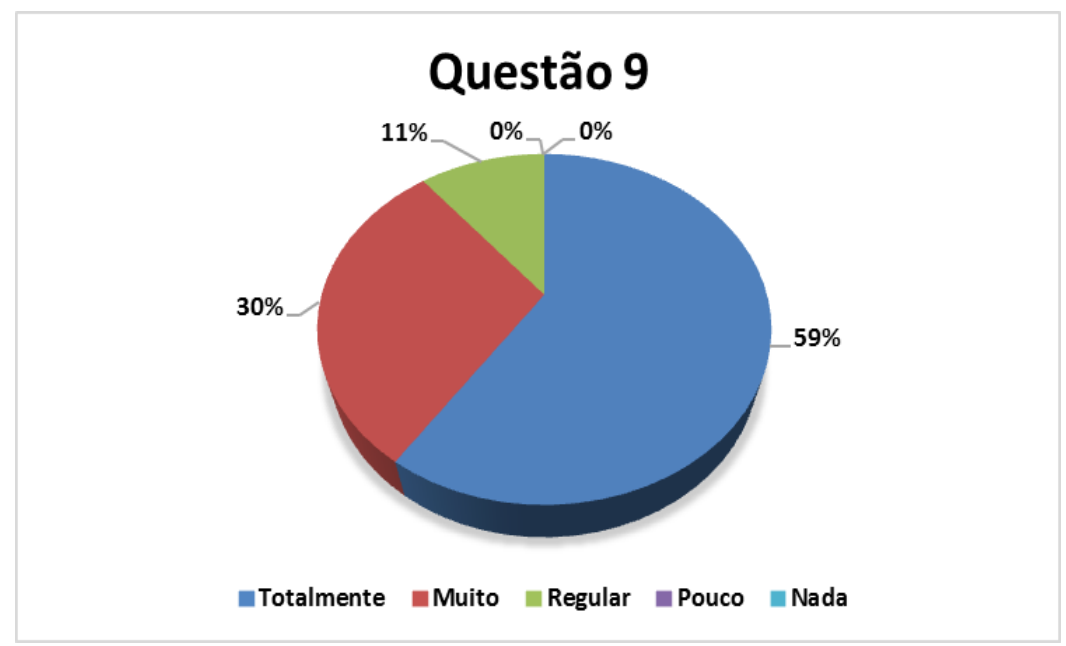

Figura 2 - Adoção de ferramentas similares para outras disciplinas segundo a visão dos participantes do estudo. 


\section{CBIE-LACLO 2015}

Anais do XXVI Simpósio Brasileiro de Informática na Educação (SBIE 2015)

A última questão investigada apenas reforça os dados sobre a eficácia da ferramenta no processo de ensino e aprendizagem da UML. Neste âmbito, 26 alunos $(70 \%)$ responderam que a ferramenta foi eficaz. Por sua vez, 11 estudantes foram imparciais quanto a este quesito ao emitir opinião regular.

\section{Considerações Finais}

Esta pesquisa apresentou detalhes inerentes ao desenvolvimento de uma ferramenta para apoio ao ensino da UML em uma aplicação educacional aberta baseada em recursos hipermídia.

A partir dos recursos oferecidos pela aplicação da ferramenta REA-UML foi possível verificar que o ensino da UML pode ser explorado utilizando métodos diferenciados em sala de aula e por meio de atividades extraclasse que vão além dos livros didáticos tradicionais habitualmente adotados. Acrescenta-se ainda o fato de que ao explorar diferentes categorias de mídias, a ferramenta pode proporcionar maiores ganhos na aprendizagem de estudantes que, possivelmente, apresentam diferentes estilos de aprendizagem.

Os resultados observados a partir da avaliação da ferramenta constatam que, de modo geral, houve aprovação dos estudantes com relação ao uso do REA-UML. Os comentários relatados pelos participantes da pesquisa corroboram o resultado por meio de inúmeras opiniões positivas. Em contrapartida, a maioria das críticas relatadas se refere a dois aspectos principais: falta de exercícios propostos na ferramenta e a ideia de que o REA-UML forneça um local para que o estudante possa realizar a modelagem de diagramas. No entanto, vale ressaltar que o objetivo principal da ferramenta é oferecer uma alternativa de fácil acesso ao aprendiz para o estudo da UML, e não se apresentar como uma ferramenta de modelagem devido à comprovada existência de variadas ferramentas eficientes e disponíveis na literatura para esta finalidade.

\section{Referências}

Bera, P. (2012). Analyzing the cognitive difficulties for developing and using UML class diagrams for domain understanding. Journal of Database Management, v. 23, n. 3, p. $1-29$.

Booch, G.; Rumbaugh, J.; Jacobson, I. (2012). UML: guia do usuário. Rio de Janeiro: Elsevier, 521p.

Cera, M. C.; Forno, M. H. D.; Vieira, V. G. (2012). Uma proposta para o ensino de engenharia de software a partir da resolução de problemas. Revista Brasileira de Informática na Educação, v. 20, n. 3, p. 116-132.

Flint, S.; Gardner, H., Boughton, C. (2004). Executable/translatable UML in computing education. Proceedings of the Sixth Australasian Conference on Computing Education, v. 30, p. 69-75.

Furgeri, S. (2013). Modelagem de sistemas orientados a objetos. São Paulo: Érica, $304 p$.

Grützner, I.; Bunse, C. (2002). Teaching object-oriented design with UML - a blended learning approach. Proceedings of $16^{\text {th }}$ European Conference on Object-Oriented Programming - ECOOP 2002, Spain.

Guedes, G. T. A. (2011). UML 2: uma abordagem prática. São Paulo: Novatec Editora, $484 p$. 


\section{CBIE-LACLO 2015}

Anais do XXVI Simpósio Brasileiro de Informática na Educação (SBIE 2015)

Kirner, C. (2013). A educação permeando a tecnologia em aplicações educacionais abertas baseadas em hipermídia e realidade aumentada. Revista Contemporaneidade, Educação e Tecnologia, v. 1, n. 3, p. 76-87.

Kuzniarz, L.; Börstler, J. (2011). Teaching modeling - an initial classification of related issues. Proceedings of the $7^{\text {th }}$ Educators'Symposium @ Models 2011: Software Modeling in Education, v. 52.

Larman, C. (2007). Utilizando UML e padrões. Porto Alegre: Bookman, 696p.

Luque, L. et al. (2014). Are you seeing this? What is available and how can we include blind students in virtual UML learning activities. Anais do XXV Simpósio Brasileiro de Informática na Educação - SBIE, Dourados.

Melo, A. C. (2010). Desenvolvendo aplicações com UML 2.2: do conceitual à implementação. 3 ed. Rio de Janeiro: Brasport, 320p.

Nicolaci-da-Costa, A. M. (2002). Revoluções tecnológicas e transformações subjetivas. Psicologia: teoria e pesquisa, v. 18, n. 2, p. 193-202.

Paiva, S.; Medeiros, A. (2011). ESCollab: uma metodologia colaborativa voltada para o ensino de engenharia de software. Anais do XXII Simpósio Brasileiro de Informática na Educação - SBIE, Aracaju.

Rodrigues, C. S. C. (2012). Visar3D - uma abordagem baseada em tecnologias emergentes $3 D$ para o apoio à compreensão de modelos UML. 166f. Tese (Doutorado em Engenharia de Sistemas e Computação), Universidade Federal do Rio de Janeiro, Rio de Janeiro.

Santana, B.; Rossini, C.; Pretto, N. L. (2012). Recursos educacionais abertos: práticas colaborativas e políticas públicas. Revista Teias, v. 13, n. 30, p.391-412.

Santos, A. I. (2013). Recursos educacionais abertos no Brasil: o estado da arte, desafios e perspectivas para o desenvolvimento e inovação. 1 ed. São Paulo: CETICbr.

Seabra, R. D.; Romano, V. P.; Oliveira, N. (2014). Uma abordagem no ensino de variação linguística em uma aplicação educacional aberta baseada em hipermídia. Anais do XXV Simpósio Brasileiro de Informática na Educação - SBIE, Dourados.

Silva, A. S. S. D. (2013). Uso de recurso educacional com mídias interativas e integradas on-line em ensino e aprendizagem. 120f. Dissertação (Mestrado em Ensino de Ciências) - Universidade Federal de Itajubá, Itajubá.

Silva, C. E.; Pansanato, L. T. E.; Fabri, J. A. (2010). Ensinando diagramas UML para estudantes cegos. Anais do XVIII CIESC-XXXVI CLEI, Assunção.

Tanaka, S. S. (2011). O poder da tecnologia de workflow e dos mapas conceituais no processo de ensino e aprendizagem da UML. 114f. Dissertação (Mestrado em Ciência da Computação) - Centro de Ciências Exatas, Universidade Estadual de Londrina, Londrina.

Torres, F. M. C. (2014). Trabalho colaborativo baseado em recursos educacionais abertos: uma experiência no ensino de ciências. 121f. Dissertação (Mestrado Profissional em Ensino de Ciências) - Universidade Federal de Itajubá, Itajubá. 